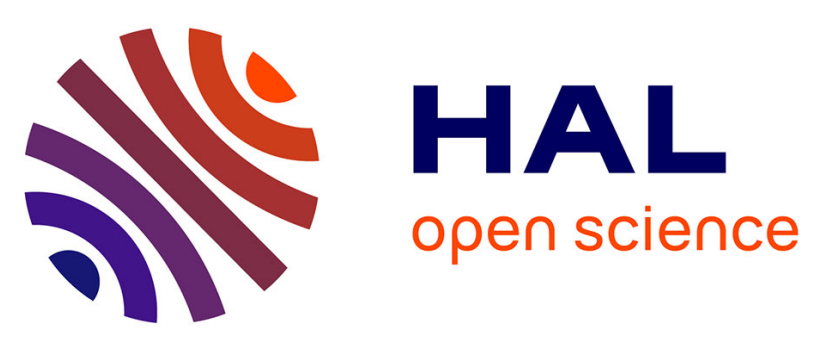

\title{
3-D Mobile-to-Mobile channel tracking with first-order autoregressive model-based Kalman filter
}

\author{
Soukayna Ghandour - Haidar, Laurent Ros, Jean-Marc Brossier
}

\section{To cite this version:}

Soukayna Ghandour - Haidar, Laurent Ros, Jean-Marc Brossier. 3-D Mobile-to-Mobile channel tracking with first-order autoregressive model-based Kalman filter. ISSPIT 2013 - IEEE International Symposium on Signal Processing and Information Technology, Dec 2013, Athènes, Greece. pp.000464000469, 10.1109/ISSPIT.2013.6781925 . hal-00986726

\section{HAL Id: hal-00986726 \\ https://hal.science/hal-00986726}

Submitted on 4 May 2014

HAL is a multi-disciplinary open access archive for the deposit and dissemination of scientific research documents, whether they are published or not. The documents may come from teaching and research institutions in France or abroad, or from public or private research centers.
L'archive ouverte pluridisciplinaire HAL, est destinée au dépôt et à la diffusion de documents scientifiques de niveau recherche, publiés ou non, émanant des établissements d'enseignement et de recherche français ou étrangers, des laboratoires publics ou privés. 


\title{
3-D Mobile-to-Mobile channel tracking with first-order autoregressive model-based Kalman filter
}

\author{
Soukayna GHANDOUR-HAIDAR, Laurent ROS, Jean-Marc BROSSIER
}

\begin{abstract}
This paper deals with channel estimation in Mobileto-Mobile communication assuming three-dimensional scattering environment. It approximates the channel by a first-order autoregressive (AR(1)) model and tracks it by a Kalman filter. The common method used in the literature to estimate the parameter of $\operatorname{AR}(1)$ model is based on a correlation matching criterion. We propose another criterion based on the Minimization of the Asymptotic Variance of the Kalman filter, and we justify why it is more appropriate for slow fading variations. This paper provides the closed-form expression of the optimal $\operatorname{AR}(1)$ parameter under minimum asymptotic variance criterion and the approximated expression of the estimation variance in output of the Kalman filter, both for Fixed-to-Mobile and Mobile-to-Mobile communication channels.
\end{abstract}

Index Terms-Channel estimation, Autoregressive model, Kalman filter, Mobile-to-Mobile communication, 3-D scattering environment.

\section{INTRODUCTION}

Different models are suitable to describe the radio-mobile communication channel, depending on the scattering environment and if only one or both terminals are in mobility. Regarding the scattering environment, one can adopt a twodimensional (2-D) scattering model, as in vehicular mobileradio reception, or a three-dimensional (3-D) scattering model, as for personal communication reception, particularly within buildings [1]. In the first case, we assume that a number of waves traveling only in the horizontal plane arise/leave the Receiver/Transmitter $\left(R_{x} / T_{x}\right)$ antenna. However, for the 3D scattering model, the scattered waves may propagate by diffraction from the edges of buildings down to the street and, thus, not necessarily travel horizontally. That is the reason to adopt a 2-D scattering model for rural environments and a 3-D scattering model for urban environments [2]. A 3-D scattering model is appropriate if the transmitted/received signal from the terminal arises/leaves from/for any direction with equal probability and the $R_{x} / T_{x}$ has an isotropic response [1].

Regarding the mobility, this radio link can be a Fixed-toMobile (F-to-M) or a Mobile-to-Mobile (M-to-M) communication channels. The first case is seen with cellular communications, where the base station (BS) is fixed at a high altitude, receives the signal within a narrow beam-width, and the mobile station (MS) is surrounded by local scatterers. This is the so-called typical macro-cell [3].

However, M-to-M communication channels have recently received much attention. They are expected to play an impor-

The authors are with GIPSA-Lab, Image and Signal Department, BP46, 38402 Saint-Martin d'Héres, France, (email:soukayna.ghandour@gipsalab.grenoble-inp.fr, laurent.ros@gipsa-lab.grenoble-inp.fr, jeanmarc.brossier@gipsa-lab.grenoble-inp.fr). tant role in many new applications, such as Mobile Ad-hoc NETworks (MANETs), Private Mobile Radio systems, i.e. Terrestrial Trunked Radio (TETRA) with direct mode operation (DMO), intelligent transportation systems for dedicated short range communications where the communication links must be extremely reliable, relay-based cellular networks, and intervehicular communications, i.e. safety, traffic efficiency, and infotainment mobile applications. In this case, both the $R_{x}$ and the $T_{x}$ are normally surrounded by local scatterers [2]-[6]. The problem of channel estimation has been treated in several contexts, but the performance analysis of estimation algorithms in case of 3-D scattering model or M-to-M communication channel case is recent. To facilitate the design of an estimation algorithm, an approximated recursive linear model is simpler than the real channel variation model. In this perspective, an autoregressive model at order $p(\operatorname{AR}(\mathrm{p}))$ can be used. In many papers (see details and references in [7] and [8]), the AR(p) coefficients calculation for a given normalized Doppler frequency $\left(f_{d} T\right)$ is based on a Correlation Matching (CM) criterion. This CM criterion imposes that the autocorrelation coefficients of the approximated autoregressive process perfectly match the sampled autocorrelation function of the true Channel Gain (CG) for $p$ lags [13], [18]. The hint that leads to use the $\mathrm{CM}$ criterion derives from the Yule-Walker equation adequate for the autoregressive model.

In this work, we use the first order autoregressive AR(1) approximation as it has been widely used in various wireless communication systems ( [8], [16], [17], [18]). We extend our previous work in [7] to the case of 3-D model and to both F-to-M and M-to-M cases, and we deal with the choice of the AR(1) coefficient required for improving the standard $\mathrm{AR}(1)$ model tuning. In fact, the CM coefficient tuning method may lead to poor performance for $p=1$, in the case of slow fading Rayleigh channel with Jakes' spectrum, compared to other methods as pointed out in [8], [7], and [9] for the one link scenario and in [10] for the dual link scenario. Then, the AR(1) parameter can be tuned from another criterion like the minimization of the steady-state estimation error variance, which is called the Minimum Asymptotic Variance (MAV). This method is more effective than the CM method for a Rayleigh Jakes' channel, for both one-link [7] and Amplifyand-Forward Relay channel [10]. In the first study [7], we provide analytic results and a closed-form expression for the optimum $\operatorname{AR}(1)$ parameter and the associated mean square error $(M S E)$ for a given one link channel state $\left(f_{d} T, S N R\right)$.

To get an online real time estimation of the true channel, we can various filters, i.e. Kalman Filter (KF), particle filters, linear MMSE estimator or least square estimator. In this study, 
we keep using the KF to track the channel, based on an AR(1) approximation model following the literature and as in our previous works [7] [10]. In a linear Gaussian problem, the KF minimizes the MSE of the estimated parameters when the KF state model closely matches the real system [11].

The paper is organized as follows: we first write the system model used to represent the mobile channel with 3-D scattering environment in section II. In section III, we provide a general theoretical frequency-domain analysis of the estimation error in terms of static and dynamic contributions. We use this analytic approach, for given Source and Destination Doppler frequencies and for a given Signal-to-Noise Ratio $(S N R)$, to derive original approximate closed-form expressions of the optimal AR(1) coefficient and of the corresponding MSE under MAV criterion. These results are validated by simulation in section IV. Section V concludes.

\section{SySTEM MODEL AND OBJeCTIVES}

\section{A. Observation and State Models}

The discrete-time observation equation $r_{k}$ at the destination $D$ is:

$$
r_{k}=s_{k} \alpha_{k}+w_{k}
$$

- $s_{k}$ is the transmitted symbol from the source $S$ at symbol time index $k$. The modulation alphabet of the symbol can be Phase-Shift-Keying or Quadrature-AmplitudeModulation. We assume $\left\{s_{k}, k \in \mathbb{Z}\right\}$ is a zero-mean white sequence with variance $\sigma_{s}^{2}$. This sequence can be known (pilot-aided mode), or unknown (data-aided mode).

- $\alpha_{k}$ is a zero-mean circular complex CG with variance $\sigma_{\alpha}^{2}$. The sequence $\left\{\alpha_{k}, k \in \mathbb{Z}\right\}$ is a stationary narrow-band process with Power Spectral Density (PSD) $\Gamma_{\alpha}(f)$.

- $w_{k}$ is the zero-mean additive white circular complex Gaussian noise at the destination $D$ with variance $\left(\sigma_{w}\right)^{2}$

For theoretical analysis, we suppose the symbols are known (pilot-aided mode) $)^{1}$ or perfectly decided. The effect of decision error on the Bit-Error-Rate (BER) will be tested in simulation section. The observation equation (1) can be then normalized to the new observation equation

$$
y_{k}=\frac{r_{k}}{s_{k}}=\alpha_{k}+n_{k}
$$

With $n_{k}$ is a white noise with variance $\sigma_{n}^{2}=\sigma_{w}^{2} \cdot K_{\text {mod }}$ where $K_{\text {mod }}=\mathbb{E}\left\{\left|\frac{1}{s_{k}}\right|^{2}\right\}$ can be computed for a given modulation scheme ( $\mathbb{E}$ denotes the expectation). The $S N R$ after this normalization is:

$$
S N R=10 \log _{10} \frac{\sigma_{\alpha}^{2}}{\sigma_{n}^{2}}=S N R_{\text {in }}-10 \log _{10}\left[K_{\text {mod }} \sigma_{s}^{2}\right]
$$

where $S N R_{i n}=10 \log _{10} \frac{\sigma_{s}^{2} \sigma_{\alpha}^{2}}{\sigma_{w}^{2}}$ is the $S N R$ at the Destination (before normalization).

- For a 3-D F-to-M communication channel [1]:

- The CG has a circular complex Gaussian Probability Density Function (PDF). This propagation model is called single Rayleigh link.

\footnotetext{
${ }^{1}$ As for example, in an OFDM system through multipath frequency selective channel, where we have to track the CG for each pilot subcarrier [14].
}

- The Doppler spectrum $\Gamma_{\alpha}(f)$ is uniform out to the maximum positive and negative Doppler shifts $f_{\max }=f_{d}$ centered around $0 \mathrm{~Hz}$ in the Baseband representation. The temporal autocorrelation function for lag $m, R_{\alpha}[m]=\mathbb{E}\left\{\alpha_{k} \alpha_{k-m}^{*}\right\}$, is then a sinc function, where $\operatorname{sinc}(x)=\frac{\sin (x)}{x}$ and $\operatorname{sinc}(0)=1$.

- In general M-to-M cases, especially in indoor and microcellular propagation studies, the propagation model is not a Single Rayleigh link, a more realistic propagation model is the cascaded Rayleigh links [5]. This scenario assumes partial signal combining on all the scatterers instead of grouped scatterers around the mobile terminals. In this case, the situation is similar to a succession of MobileFixed and Fixed-Mobile Rayleigh links, then:

- The PDF is no longer Gaussian, but a modified Bessel function of the second kind of zero order [5], [15], i.e. the distribution of the product of two Circular Complex Gaussian variables.

- The Doppler spectrum designed by $\Gamma_{\alpha}$ is a convolution of two uniform spectra. The expression and shape of $\Gamma_{\alpha}(f)$ are given in Table I and figure 1 respectively. The normalized spectrum support $f_{\max } T=f_{S} T+f_{D} T$, where $f_{S}$ and $f_{D}$ are the source and destination Doppler frequencies respectively and $T$ is the symbol period. The autocorrelation function is then the product of two sinc function [1], [12]: $R_{\alpha}[k]=\operatorname{sinc}\left(2 \pi f_{S} k\right) \operatorname{sinc}\left(2 \pi f_{D} k\right)$.

In the perspective to use KF, a state-space formulation of the CG dynamic is required. We approximate the time-varying CG $\alpha_{k}$ by an $\operatorname{AR}(1)$ model $\tilde{\alpha}_{k}$ :

$$
\tilde{\alpha}_{k}=a \cdot \tilde{\alpha}_{k-1}+e_{k}
$$

where $e_{k}$ is the white circular complex Gaussian state noise with variance $\sigma_{e}^{2}$. The variances of the approximated and real processes are tuned to be the same, $\sigma_{\tilde{\alpha}}^{2}=\sigma_{\alpha}^{2}$ (i.e. $R_{\tilde{\alpha}}[0]=$ $\left.R_{\alpha}[0]\right)$. Therefore, the state noise variance is:

$$
\sigma_{e}^{2}=\left(1-a^{2}\right) \sigma_{\alpha}^{2}
$$

\section{B. Tuning the AR(1) coefficient}

It is straightforward from the Yuke Walker equations for autoregressive model, that the AR(1) coefficient $a$ verifies

$$
a=\frac{R_{\tilde{\alpha}}[1]}{R_{\tilde{\alpha}}[0]}
$$

where $R_{\tilde{\alpha}}[0]$ and $R_{\tilde{\alpha}}[1]$ are the two first autocorrelation coefficients of the $\operatorname{AR}(1)$ approximated process $\tilde{\alpha}$. The remaining issue is to fix $R_{\tilde{\alpha}}[1]$ using the normalization $R_{\tilde{\alpha}}[0]=R_{\alpha}[0]$. In this paper, we refer to two main criteria:

1) The CM criterion [13]. In this case, the correlation coefficients of the AR(1) process $\tilde{\alpha}$ coincide with the exact CG correlation coefficients (i.e. $R_{\tilde{\alpha}}[0]=R_{\alpha}[0]$, and $\left.R_{\tilde{\alpha}}[1]=R_{\alpha}[1]\right)$. We denote by $a_{C M}$ the $\mathrm{AR}(1)$ coefficient under $\mathrm{CM}$ criterion.

2) The MAV criterion [8] [7]. In this case, the coefficient of the $\operatorname{AR}(1)$ process is calculated in order to minimize 
Figure 1. Doppler PSD for different cases. In the F-to-M case, $f_{d}$ is the Doppler frequency of the mobile terminal. In the $M$-to-M case with triangular shape, the source and destination move at same velocity, with corresponding Doppler frequency $f_{d}$. In the M-to-M case with trapezoïdal spectrum shape, $f_{S}$ and $f_{D}$ are the source and destination Doppler frequencies respectively. Without loss of generality, we suppose here that the destination moves at a velocity higher than the velocity of the source.
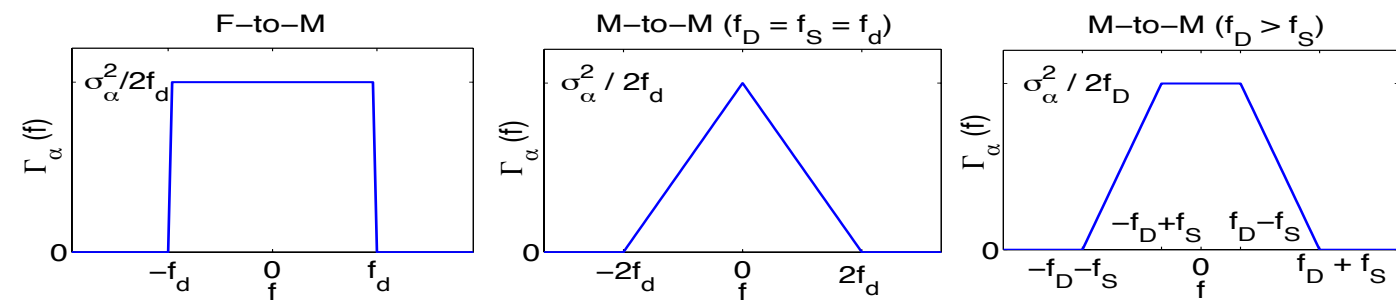

Table I

Expression of Doppler PSD and the corresponding value of $I_{\alpha}$ for different cases.

\begin{tabular}{|c|c|c|c|}
\hline case & & $\Gamma_{\alpha}(f)$ & $I_{\alpha}$ \\
\hline F-to-M & & $\frac{\sigma_{\alpha}^{2}}{2 f_{d}} \quad-f_{d}<f<+f_{d}$ & $\frac{\sigma_{\alpha}^{2}}{3}\left(2 \pi f_{d} T\right)^{2}$ \\
\hline M-to-M $\left(f_{D}=f_{S}=f_{d}\right)$ & & $\frac{\sigma_{\alpha}^{2}}{\left(2 f_{d}\right)^{2}}\left\{-|f|+2 f_{d} \quad\right.$ if $\quad 0<|f|<2 f_{d}$ & $\frac{2 \sigma_{\alpha}^{2}}{3}\left(2 \pi f_{d} T\right)^{2}$ \\
\hline M-to-M $\left(f_{D}>f_{S}\right)$ & $\frac{\sigma_{\alpha}^{2}}{4 f_{S} f_{D}}$ & $\begin{array}{rcc}2 f_{S} & \text { if } & 0<|f|<f_{D}-f_{S} \\
-|f|+f_{D}+f_{S} & \text { if } & f_{D}-f_{S}<|f|<f_{D}+f_{S}\end{array}$ & $\frac{\sigma_{\alpha}^{2}}{3}\left[\left(2 \pi f_{S} T\right)^{2}+\left(2 \pi f_{D} T\right)^{2}\right]$ \\
\hline
\end{tabular}

the error variance in asymptotic regime. We denote by $a_{M A V}$ the $\mathrm{AR}(1)$ coefficient under MAV criterion.

One contribution of this paper is to find the analytical expression of $a_{M A V}$ wrt the statistical parameters of the channel.

\section{Kalman Filter Equations}

The simplified observation (2) is then approximated by:

$$
y_{k} \approx \tilde{\alpha}_{k}+n_{k}
$$

Given the state and observation equations (3) and (6), the Kalman equations are then reduced to (see [11]):

$$
\begin{aligned}
K_{k} & =\left[a^{2} P_{k-1}+\sigma_{e}^{2}\right] /\left[a^{2} P_{k-1}+\sigma_{e}^{2}+\sigma_{n}^{2}\right] \\
P_{k} & =\left(1-K_{k}\right)\left(a^{2} P_{k-1}+\sigma_{e}^{2}\right) \\
\hat{\alpha}_{k} & =a \hat{\alpha}_{k-1}+K_{k}\left(y_{k}-a \hat{\alpha}_{k-1}\right)
\end{aligned}
$$

where $K_{k}$ is the Kalman gain at iteration $k$ and $P_{k}$ is the estimation error variance. We denote by $\hat{\alpha}_{k}$ the on-line unbiased estimate of the true $\alpha_{k}$.

\section{MSE ANALYSIS AND OPTIMIZATION}

Since the linear system (6) and (3) is observable and controllable, an asymptotic regime is reached ( [14]). We calculate the asymptotic values of the Kalman Gain $\left(K_{\infty}\right)$ and the error variance $\left(P_{\infty}\right)$ based on (7) and (8), given $a^{2}=1-\frac{\sigma_{e}^{2}}{\sigma_{\alpha}^{2}}$ :

$$
\begin{aligned}
P_{\infty} & =\frac{\sigma_{n}^{2}\left(a^{2}-1\right)-\sigma_{e}^{2}+\sqrt{\Delta}}{2 a^{2}} \\
K_{\infty} & =\frac{a^{2} P_{\infty}+\sigma_{e}^{2}}{a^{2} P_{\infty}+\sigma_{e}^{2}+\sigma_{n}^{2}}
\end{aligned}
$$

with $\Delta=\left(\sigma_{n}^{2}+\sigma_{e}^{2}-a^{2} \sigma_{n}^{2}\right)^{2}+4 a^{2} \sigma_{n}^{2} \sigma_{e}^{2}$. We assume that $\sigma_{e}^{2}<<\sigma_{n}^{2}$ and we are working in the range of $S N R>0$ where $S N R$ is in $d B$, which means that

$$
\sigma_{e}^{2}<<\sigma_{n}^{2}<\sigma_{\alpha}^{2}
$$

Then, under this assumption, we can approximate the expression of the asymptotic Kalman Gain (See [7])

$$
K_{\infty} \approx \frac{\sigma_{e}}{\sigma_{n}}
$$

For the asymptotic regime, we use the Z-Transform ( $Z$ ) of equation (9), then:

$$
\begin{aligned}
\hat{\alpha}(z) & =L(z) Y(z) \\
L(z) & =\frac{K_{\infty} z}{z-a\left(1-K_{\infty}\right)} \\
\varepsilon(z) & =[1-L(z)] \alpha(z)-L(z) N(z)
\end{aligned}
$$

where $L(z)$ is the steady-state transfer function of the KF, $\varepsilon(z)=z(\varepsilon(k))$, and $\varepsilon(k)=\alpha_{k}-\hat{\alpha}_{k}$. The error is composed from two parts, the dynamic error related to $\alpha_{k}$ and the static error related to $n_{k}$ :

$$
\begin{aligned}
M S E & =\mathbb{E}\left\{|\varepsilon(k)|^{2}\right\}=M S E_{1}+M S E_{2} \\
M S E_{1} & =\int_{-f_{\max }}^{+f_{\max }}\left|1-L\left(e^{j 2 \pi f T}\right)\right|^{2} \Gamma_{\alpha}(f) \mathrm{d} f \\
M S E_{2} & =\int_{\frac{-1}{2 T}}^{\frac{1}{2 T}}\left|L\left(e^{j 2 \pi f T}\right)\right|^{2} \Gamma_{n}(f) \mathrm{d} f
\end{aligned}
$$

where $\Gamma_{\alpha}(f)$ is the CG spectrum as defined in table I, and $\Gamma_{n}(f)$ is the PSD of the noise $n_{k}$. We assume that $z^{-1}=e^{-j 2 \pi f T} \approx 1-j 2 \pi f T$ for low normalized frequency range, i.e. for $f T<f_{d} T<<1$, using (13), $\left|1-L\left(e^{j 2 \pi f T}\right)\right|^{2} \approx$ $\left|\frac{2 \pi f_{V L F} T+j 2 \pi f T}{2 \pi f_{c} T+j 2 \pi f T}\right|^{2}$, where, according to [7]:

- $2 \pi f_{V L F} T=\frac{1}{a}-1=\left[1-\frac{\sigma_{e}^{2}}{\sigma_{\alpha}^{2}}\right]^{-\frac{1}{2}}-1 \approx \frac{1}{2} \frac{\sigma_{e}^{2}}{\sigma_{\alpha}^{2}}($ using (4)) 
- $2 \pi f_{c} T=\frac{1-a\left(1-K_{\infty}\right)}{a\left(1-K_{\infty}\right)} \approx \frac{\sigma_{e}}{\sigma_{n}}$ (using (11))

Then $1-L\left(e^{j 2 \pi f T}\right)$ is mainly a high-pass filter with upper cutoff frequency $f_{c}$, and with another very low cut-off frequency $f_{V L F}$ (see Figure 3 in [7]), but with a negligible effect on the $M S E$ computation. As seen in [7], only the medium asymptote is useful for the $M S E_{1}$ computation, i.e. $\left|1-L\left(e^{j 2 \pi f T}\right)\right|^{2} \approx$ $\left|\frac{2 \pi f T}{2 \pi f_{c} T}\right|^{2} \approx \frac{\sigma_{n}^{2}}{\sigma_{e}^{2}}|2 \pi f T|^{2}$. Then

$$
\begin{aligned}
M S E_{1} & \approx \frac{\sigma_{n}^{2}}{\left(1-a^{2}\right) \sigma_{\alpha}^{2}} \int_{-f_{\max }}^{+f_{\max }}(2 \pi f T)^{2} \Gamma_{\alpha}(f) \mathrm{d} f \\
& \approx \frac{\sigma_{n}^{2}}{\left(1-a^{2}\right) \sigma_{\alpha}^{2}} I_{\alpha}
\end{aligned}
$$

where

$$
I_{\alpha}=\int_{-f_{\max }}^{+f_{\max }}(2 \pi f T)^{2} \Gamma_{\alpha}(f) \mathrm{d} f
$$

$n_{k}$ is a discrete-time additive white Gaussian noise,

$$
\Gamma_{n}(f)=\sigma_{n}^{2} T \quad \text { for } \quad \frac{-1}{2 T}<f<\frac{1}{2 T}
$$

Then using (13) and (20), we calculate $M S E_{2}$ and obtain (see appendix A):

$$
M S E_{2}=\frac{K_{\infty}^{2} \sigma_{n}^{2}}{1-a^{2}\left(1-K_{\infty}\right)^{2}}
$$

Given equation (4), the approximated expression of $K_{\infty}$, and under the assumption $\sigma_{e}^{2}<<\sigma_{n}^{2}$, we have:

$$
M S E_{2} \approx \sigma_{n}^{2} \frac{\sigma_{e}}{2 \sigma_{n}}=\frac{\sqrt{\left(1-a^{2}\right)} \sigma_{n} \sigma_{\alpha}}{2}
$$

From (15), (18) and (22), it is easy to write $M S E$ as a function of the parameter $a$ :

$$
\operatorname{MSE}(a)=\frac{\Omega}{1-a^{2}}+\beta \sqrt{1-a^{2}}
$$

where $\Omega=\frac{\sigma_{n}^{2}}{\sigma_{\alpha}^{2}} I_{\alpha}$ and $\beta=\frac{\sigma_{n} \sigma_{\alpha}}{2}$.

We propose to find the AR(1) coefficient $\left(a_{M A V}\right)$ that gives the minimum asymptotic estimation error variance. This verifies: $\frac{d M S E}{d a}=0$ and then $\left(1-a_{M A V}^{2}\right)^{\frac{3}{2}}=\frac{2 \Omega}{\beta}$.

The AR(1) coefficient $a_{M A V}$ chosen under the MAV criterion and the expression of the resulting minimum asymptotic $M S E_{M A V}=\operatorname{MSE}\left(a_{M A V}\right)$ are then given as a function of $I_{\alpha}$ (which depends on the Doppler PSD via the integral (19)):

$$
\begin{aligned}
a_{M A V} & =\sqrt{1-\left[\frac{2 \Omega}{\beta}\right]^{\frac{2}{3}}}=\sqrt{1-\sqrt[3]{\frac{16 \sigma_{n}^{2} I_{\alpha}^{2}}{\sigma_{\alpha}^{6}}}} \\
M S E_{M A V} & =\Omega\left[\frac{\beta}{2 \Omega}\right]^{\frac{2}{3}}+\beta\left[\frac{2 \Omega}{\beta}\right]^{\frac{1}{3}}=\frac{3}{2}\left[\frac{\sigma_{n}^{4} I_{\alpha}}{2}\right]^{\frac{1}{3}}
\end{aligned}
$$

We consider three cases: F-to-M and M-to-M with same or different velocities. We need to find the overall channel spectrum in each case, and the corresponding $I_{\alpha}$, in order to know the value of the dynamic error. The calculations are given in the appendix $\mathrm{B}$. We high that

$$
I_{\alpha}=\frac{\sigma_{\alpha}^{2}\left(2 \pi f_{e q} T\right)^{2}}{3} \text { where } f_{e q}^{2}=f_{S}^{2}+f_{D}^{2}
$$

We replace the expression of $I_{\alpha}$ given by (26) in (24) and (25). We have then:

$$
\begin{gathered}
a_{M A V}=\sqrt{1-\sqrt[3]{\frac{16}{9} \frac{\sigma_{n}^{2}}{\sigma_{\alpha}^{2}}\left(2 \pi f_{e q} T\right)^{4}}} \\
M S E_{M A V}=\sqrt[3]{\frac{9}{16} \sigma_{n}^{4} \sigma_{\alpha}^{2}\left(2 \pi f_{e q} T\right)^{2}}
\end{gathered}
$$

\section{Simulation Results}

We present Monte Carlo simulations for three cases: F-to$M$ and $M-$-to-M with same and different velocities. Excepted for the BER results (Figure 5), we consider pilot-aided mode, and then the results are independent on the modulation scheme according to observation equation (2)

\section{A. F-to-M case}

The Doppler spectrum is flat, and the autocorrelation function used to calculate $a_{C M}$ is a Sinc function. The results are shown in Figure 2. We compare the empirical MSE obtained (under CM and MAV criteria) to the theoretical value of $M S E$ under MAV criterion (28). MSE is plotted as a function of Doppler frequencies for different values of $S N R$.

With CM criterion, the MSE is approximately constant with respect to the Doppler frequency and is equivalent to $\sigma_{n}^{2}$ for $S N R=10 \log _{10}\left(\frac{\sigma_{\alpha}^{2}}{\sigma_{n}^{2}}\right)>10 \log _{10}(2) \approx 3 d B$. This corroborates the results of [7] obtained for the 2-D scattering model.

Under MAV criterion, the theoretical value $M S E_{M A V}$ seems to be approximately the same as the MSE computed by Monte Carlo simulation (denoted by $M A V$ in the legend) for all the usual range of $S N R$ between 0 and $20 \mathrm{~dB}$, so we validate the closed-form expression (28), with here $f_{e q}=f_{d}$.

Figure 2. MSE comparison of the AR(1) KF estimator based on the literature $C M$ criterion or on the MAV criterion, for different SNR, in the case of Flat Doppler Spectrum, as functions of $f_{d} T$.

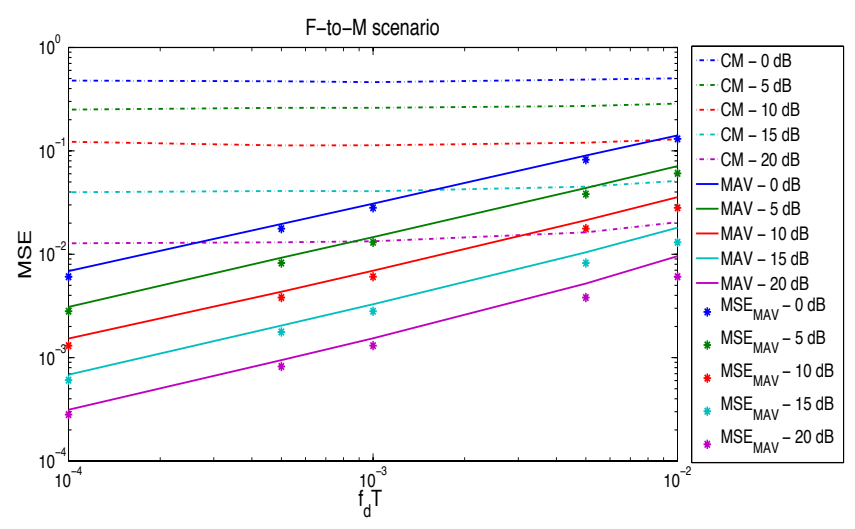




\section{B. M-to-M with equal velocities}

The Doppler spectrum is triangular. The results are shown in Figure 3. There is always an amelioration in terms of $M S E$ with the MAV criterion compared to the CM criterion. We validate the closed-form expression (28) in this case too, but we have here $f_{e q}=\sqrt{2} f_{d}$. The same remarks are observed, which is normal because we have the same formula wrt $f_{\text {eq }}$. The curves of MSE in this figure are then the same as the curves in Figure 2, but with ordinate axis shift due to the shift $\sqrt{2} f_{d}$ vs $f_{d}$ in the $M S E_{M A V}$ expression. This means that for given $f_{d}, M S E_{M A V}$ is multiplied by $2^{\frac{1}{3}}$ equivalent to $10 \log \left(2^{\frac{1}{3}}\right) \approx 1 d B$.

Figure 3. MSE comparison of the AR(1) KF estimator based on the literature $C M$ criterion or on the MAV criterion, for different SNR, in the case of Triangle Doppler Spectrum, as functions of $f_{d} T$.

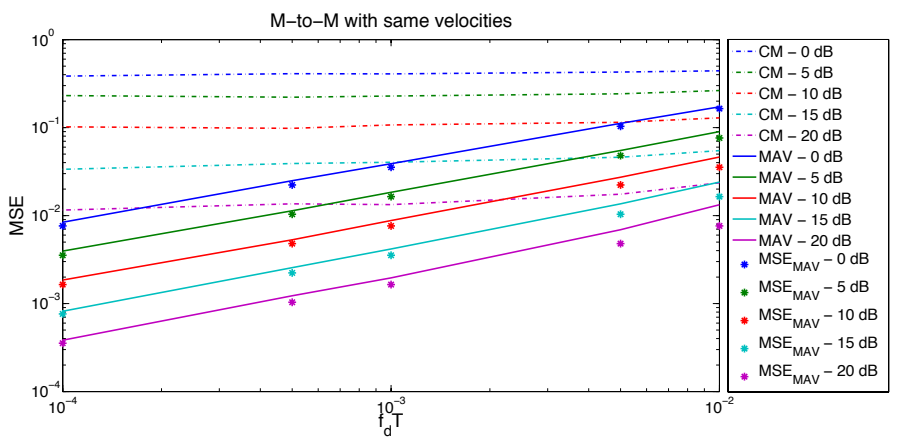

\section{M-to-M with different velocities case}

The Doppler Spectrum is trapezoïdal. The MSE behavior is the same as for a triangular Doppler Spectrum. In figure 4, $M S E_{C M}$ seems to be constant wrt $f_{e q}$ and independent of $f_{D}$ and $f_{S}$. We see that $M S E_{M A V}$ is dependent on $f_{e q} T$ only and not $f_{S} T$ or $f_{D} T$. This can be justified from (28).

Table II shows the values of $a_{C M}$ versus $a_{M A V}$. As $a_{M A V}$

Figure 4. MSE comparison of the AR(1) KF estimator based on the literature $C M$ criterion or on the MAV criterion when $f_{e q}$ is the same but $f_{D}$ and $f_{S}$ values change.

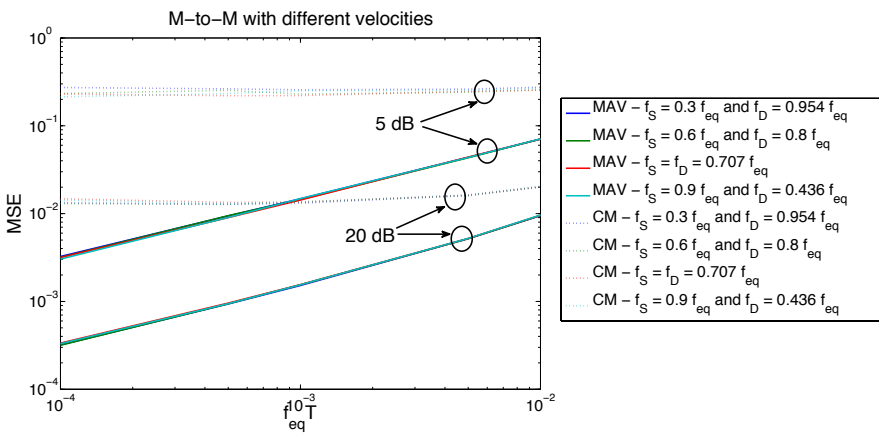

increases wrt SNR (it decreases with $\sigma_{n}^{2}$ ), and as $a_{M A V}$ increases wrt $f_{e q} T$, we see that $a_{M A V}<a_{C M}$ in the range of our assumptions. This corroborates the results shown in [10] for the 2-D dual link scenario.

In order to look at the consequences of our choice of $\mathrm{AR}(1)$ coefficient, we compare the BER obtained under CM and MAV criteria with $f_{D} T=10^{-4}$ and $f_{S} T=10^{-3}$. We consider
Figure 5. BER versus SNR for the AR(1) KF estimator based on the CM and $M A V$ criteria, for $f_{S} T=10^{-4}$ and $f_{D} T=10^{-3}$, in the case of Trapezoïdal Doppler Spectrum, with TDM training scenario

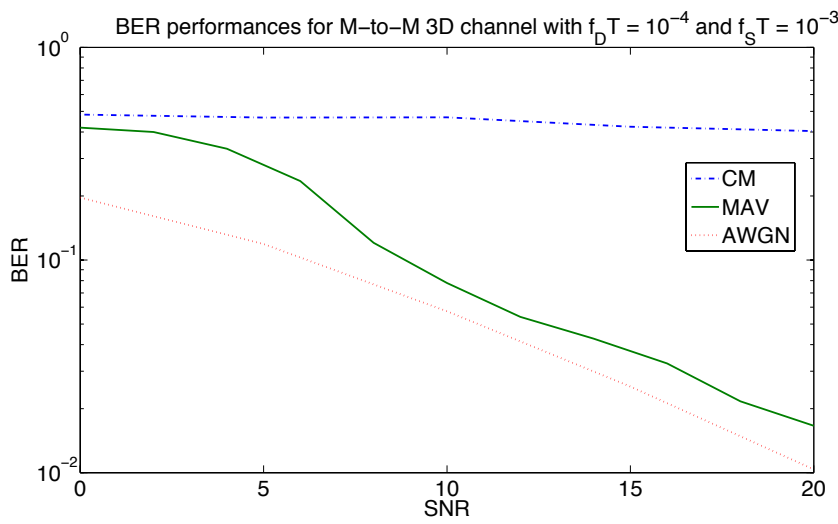

then a half-blind mode where 10 pilot symbols (known at the receiver) every 100 symbols are placed in the transmitted sequence. We use Binary Phase Shift Keying (BPSK) modulation for the normalized symbols $\left(s_{k} \in\{-1 ;+1\}\right)^{2}$, then $K_{\text {mod }}=1$, $\sigma_{s}^{2}=1$, and $S N R=S N R_{i n}$. Figure 5 shows the behavior in this case. We see significant improvement after channel estimation with MAV-KF versus the CM-KF, for a wide range of $S N R$ which corresponds to the range of $\sigma_{n}^{2}$ valid for our assumption.

\section{CONCLUSION}

This paper addresses the problem of estimating a radiomobile channel using a first-order AR(1) model-based KF, assuming a 3-D scattering environment. We calculate the overall channel spectrum depending on the status of the source and destination (F-to-M and M-to-M cases). We show by simulations that the application of the $\mathrm{CM}$ criterion in order to choose the $\mathrm{AR}(1)$ coefficient is not accurate for low SNR and low Doppler frequencies. Therefore, we switch to an MAV criterion (already proposed in [7]) to carry out the optimization of the AR(1) model. We provide an approximate expression of the MSE, and of the AR(1) (MAV) parameter for a given $S N R$ and Doppler scenario. This paper demonstrates that the $M S E$ of the AR(1) KF (MAV) is proportional to the (2/3) power of the product $\sigma_{n}^{2}\left(2 \pi f_{e q} T\right)$, where $\sigma_{n}^{2}$ is the total observation noise variance, and $f_{e q}$ is the equivalent Doppler frequency, such that $f_{e q}^{2}=f_{S}^{2}+f_{D}^{2}$.

\section{APPENDIX}

\section{A. Calculation of $\mathrm{MSE}_{2}$}

Using (13) and (20)

$$
M S E_{2}=\left(K_{\infty}^{2} \sigma_{n}^{2} T\right) \int_{\frac{-1}{2 T}}^{\frac{1}{2 T}} \frac{\mathrm{d} f}{1+A^{2}-2 A \cos (2 \pi f T)}
$$

${ }^{2}$ In this half-blind mode, in the third KF equation (9), we use $y_{k}=\frac{r_{k}}{\hat{s}_{k \mid k-1}}$ instead of equation (2), where $\hat{s}_{k \mid k-1}=s_{k}$ if $s_{k}$ is known (pilot) and $\hat{s}_{k \mid k-1}=$ $\operatorname{sgn}\left\{\Re\left(\hat{\alpha}_{k-1}^{*} \times r_{k}\right)\right\}$ if $s_{k}$ is unknown (data) (sgn is the sign function). In this case, $\hat{s}_{k \mid k-1}$ represents the a priori decision, and the final decision will be $\hat{s}_{k}=\operatorname{sgn}\left\{\Re\left(\hat{\alpha}_{k}^{*} \times r_{k}\right)\right\} .\left(^{*}\right)$ is the transpose conjugate operator. 
Table II

Numerical values of $a_{C M}=\operatorname{sinc}\left(2 \pi f_{S} T\right) \operatorname{sinc}\left(2 \pi f_{D} T\right)$ and of $a_{M A V}$ given in (27), for the $M$-to-M case with different velocities. In scenario $1, f_{S}=0.3 f_{\text {eq, }}$, in scenario $2, f_{S}=f_{D}=\frac{f_{\text {eq }}}{\sqrt{2}}$, in scenario $3, f_{S}=0.6 f_{\text {eq }}$, and in scenario $4, f_{S}=0.9 f_{\text {eq }}$. For a given $f_{\text {eq }}$, the value of $a_{C M}$ depends on $f_{D}$ and $f_{S}$ but not on the SNR, while for $a_{M A V}$, it is the opposite.

\begin{tabular}{|c|c|c|c|}
\hline & $f_{e q} T=0.0005$ & $f_{e q} T=0.001$ & $f_{e q} T=0.005$ \\
\hline$a_{C M}$ scenario 1 & 0,999998355066833 & 0,999993420278139 & 0,999835515596909 \\
\hline$a_{C M}$ scenario 2 & 0,999998355066994 & 0,999993420280710 & 0,999835517204046 \\
\hline$a_{C M}$ scenario 3 & 0,999998355067016 & 0,999993420281050 & 0,999835517416166 \\
\hline$a_{C M}$ scenario 4 & 0,999998355066912 & 0,999993420279386 & 0,999835516376127 \\
\hline$a_{M A V}$ at 5dB & 0,999810109358414 & 0,999521436486264 & 0,995900917333125 \\
\hline$a_{M A V}$ at 20dB & 0,999939955205278 & 0,999848689693471 & 0,998705575182885 \\
\hline
\end{tabular}

where $A=a\left(1-K_{\infty}\right)$. We use a variable change $u=\tan (\pi f T)$

$$
\begin{aligned}
M S E_{2} & =\frac{K_{\infty}^{2} \sigma_{n}^{2}}{\pi} \int_{-\infty}^{\infty} \frac{d u}{\left(1+A^{2}\right)\left(1+u^{2}\right)-2 A\left(1-u^{2}\right)} \\
& =\frac{K_{\infty}^{2} \sigma_{n}^{2}}{\pi(1-A)^{2}} \int_{-\infty}^{\infty} \frac{d u}{1+\left[\frac{1+A}{1-A}\right]^{2} u^{2}} \\
& =\frac{K_{\infty}^{2} \sigma_{n}^{2}}{\pi(1-A)^{2}} \int_{-\infty}^{\infty}\left[\frac{1-A}{1+A}\right] \frac{d s}{1+s^{2}} \\
& =\frac{K_{\infty}^{2} \sigma_{n}^{2}}{\pi\left(1-A^{2}\right)}\left[\tan ^{-1}(s)\right]_{-\infty}^{\infty} \\
M S E_{2} & =\frac{K_{\infty}^{2} \sigma_{n}^{2}}{1-A^{2}}=\frac{K_{\infty}^{2} \sigma_{n}^{2}}{1-a^{2}\left(1-K_{\infty}\right)^{2}}
\end{aligned}
$$

\section{B. Calculation of $I_{\alpha}$}

From (19) and the expression of $\Gamma_{\alpha}$ given in table I, we can calculate the value of $I_{\alpha}$. For the F-to-M case:

$$
\begin{aligned}
I_{\alpha} & =\int_{-f_{d}}^{+f_{d}}(2 \pi T)^{2} \frac{\sigma_{\alpha}^{2}}{2 f_{d}} f^{2} \mathrm{~d} f \\
& =(2 \pi T)^{2} \frac{\sigma_{\alpha}^{2}}{2 f_{d}}\left[\frac{f^{3}}{3}\right]_{-f_{d}}^{f_{d}}=\frac{\sigma_{\alpha}^{2}\left(2 \pi f_{d} T\right)^{2}}{3}
\end{aligned}
$$

For the M-to-M with same velocities case:

$$
\begin{aligned}
I_{\alpha} & =2 \int_{0}^{2 f_{d}}(2 \pi T)^{2} \frac{\sigma_{\alpha}^{2}}{\left(2 f_{d}\right)^{2}}\left(-f^{3}+2 f^{2} f_{d}\right) \mathrm{d} f \\
& =(2 \pi T)^{2} \frac{\sigma_{\alpha}^{2}}{2 f_{d}^{2}}\left[\frac{\left(2 f_{d}\right)^{4}}{12}\right]=\frac{2 \sigma_{\alpha}^{2}\left(2 \pi f_{d} T\right)^{2}}{3}
\end{aligned}
$$

For the M-to-M with different velocities case:

$$
I_{\alpha}=(2 \pi T)^{2} \frac{\sigma_{\alpha}^{2}}{4 f_{S} f_{D}}(2 \mathcal{J})
$$

with

$$
\begin{aligned}
\mathcal{J} & =\int_{0}^{\Delta f}\left(2 f^{2} f_{S}\right) \mathrm{d} f+\int_{\Delta f}^{\Sigma f} \Sigma f f^{2} \mathrm{~d} f-\int_{\Delta f}^{\Sigma f} f^{3} \mathrm{~d} f \\
& =\frac{\left(2 f_{S}-\Sigma f\right)(\Delta f)^{3}}{3}+\frac{(\Sigma f)^{4}}{3}-\frac{(\Sigma f)^{4}}{4}+\frac{(\Delta f)^{4}}{4} \\
& =\frac{(\Sigma f)^{4}}{12}-\frac{(\Delta f)^{4}}{12}=\frac{8 f_{D}^{3} f_{S}+8 f_{D} f_{S}^{3}}{12}
\end{aligned}
$$

where $\Sigma f=f_{D}+f_{S}$ and $\Delta f=f_{D}-f_{S}$. Then

$$
I_{\alpha}=\frac{\sigma_{\alpha}^{2}\left(\left(2 \pi f_{D} T\right)^{2}+\left(2 \pi f_{S} T\right)^{2}\right)}{3}
$$

\section{REFERENCES}

[1] R. H. Clarke and W. L. Khoo,"3-D Mobile Radio Channel Statistics", in IEEE Trans. on Vehicular Tech., vol. 46, no. 3, August 1997

[2] A. G. Zajié and G. L. Stüber, "Maximum Likelihood Method for MIMO Mobile-to-mobile Channel Parameter Estimation", in IEEE GLOBECOM Conference, Nov. 30 - Dec. 42008.

[3] G. Bakhshi, R. Saadat and K. Shahtalebi, "A Modified Two-Ring Reference Model for MIMO Mobile-to-Mobile Communication Channels ", in Inter. Symposium on Telecom., Aug. 2008

[4] M. Pätzold, B. O. Hogstad, N. Youssef and D. Kim, "A MIMO MobileTo-Mobile Channel Model: Part I - The Reference Model ", in 16th IEEE PIMRC Symposium, Sep. 2005.

[5] I. Z. Kovács, P. C. F. Eggers, K. Olesen and L. G. Petersen,"Investigations of Outdoor-to-Indoor Mobile-to-Mobile Radio Communication Channels ", in IEEE 56th Vehicular Tech. Conference, 2002.

[6] C. X. Wang, X. Cheng, and D. I. Laurenson," Vehicle-to-Vehicle Channel Modeling and Measurements: Recent Advances and Future Challenges", in IEEE Communication Magazine, pp. 96-103, Nov. 2009

[7] S. Ghandour-Haidar, L. Ros and J. M. Brossier,"On the use of first-order autoregressive modeling for Rayleigh Flat Fading Channel Estimation with Kalman filter", in Elsevier Signal Process., vol. 92, pp. 601-606, Feb. 2012.

[8] A. Barbieri, A. Piemontese, G. Colavolpe, "On the ARMA Approximation for Frequency Channels Described by the Clarke Model with Applications to Kalman-based Receivers", in IEEE Trans. on Wireless Comm., vol. 8, no. 2, Feb. 2009

[9] L. Ros, H. Hijazi, E. P. Simon, "Paths complex gain tracking algorithms for OFDM receiver in slowly-varying channels", in Proc. IEEE ISCCSP, Limassol, Cyprus, Mar. 2010.

[10] S. Ghandour-Haidar, L. Ros and J. M. Brossier, "Improving the tuning of First-Order Autoregressive Model for the estimation of Amplify and Forward Relay channel", in IEEE 19th International Conference on Telecommunications, Jounieh, Lebanon, Apr. 2012

[11] Steven M. Kay, Fundamentals of Statistical Signal Processing - Estimation Theory. Prentice Hall, 1993.

[12] A. S. Akki and F. Haber, "A Statistical Model of Mobile-to-Mobile Land Communication Channel", in IEEE Trans. on Vehicular Tech., vol. 35, no. 1, Feb. 1986.

[13] K. E. Baddour and N. C. Beaulieu, "Autoregressive modeling for fading channel simulation", in IEEE Trans. Wireless Comm., vol. 4, no. 4, pp. 1650-1662, Jul. 2005.

[14] G. K. Chui and G. Chen, Kalman Filtering with real-time applications, Springer-Verlag, 1987.

[15] D. Roque, C. Siclet and J. M. Brossier, "A Low-Complexity Multicarrier Scheme with LDPC Coding for Mobile-to-Mobile Environment", in IEEE Military Comm. Conference, Orlando, Florida, United States, Oct. 2012.

[16] T. Y. Al-Naffouri, "An EM-based forward-backward Kalman for the estimation of time-variant channels in OFDM", in IEEE Trans. on Signal Proc., vol. 55, Jul. 2007

[17] Z. Liu, X. Ma, and G. B. Giannakis, "Space-time coding and Kalman Filtering for time selective fading channels", in IEEE Trans. on Comm., vol. 50, no. 2, Feb. 2002.

[18] C. Komninakis, C. Fragouli, A. H. Sayed and R. D. Wesel, "Multi-Input Multi-Output fading channel tracking and equalization using Kalman estimation", in IEEE Trans. on Signal Proc., vol. 50, May 2002. 